\title{
Osteocondrosis en el toro de lidia y evaluación de su efecto sobre la movilidad del animal
}

\section{Osteochondrosis in fighting bulls and evaluation of its effect on the animal mobility}

\author{
J uan Manuel Lomillos Pérez ${ }^{\mathrm{a}^{*}}$, Marta Elena Alonso de la Varga ${ }^{\mathrm{a}}$
}

\begin{abstract}
RESUMEN
La osteocondrosis es un proceso degenerativo de las superficies articulares. Es una enfermedad descrita ampliamente en el caballo, con escasas referencias bibliográficas en bovino y prácticamente inexistentes en la raza de lidia. El objetivo de este trabajo fue informar sobre la prevalencia de osteocondrosis en toros y novillos, estudiar sus posibles causas y analizar la repercusión de esta patología en la movilidad del animal durante la lidia. Para ello se han estudiado 120 animales de la raza bovina de lidia, de 3, 4 y 5 años, lidiados en diferentes plazas de toros. Durante el faenado de las reses posterior a la lidia se realizó un estudio macroscópico de las articulaciones carpo-metacarpianas, estudiándose la presencia de lesión en las caras articulares implicadas y su grado de gravedad. Paralelamente se grabó la lidia de cada animal para el análisis de su movilidad y evaluación del síndrome de caída. Los resultados reflejan una lesión del cartílago articular en mayor o menor grado en aproximadamente el 80 \% de los animales estudiados, apareciendo en el 63 \% de los casos sobre la cara medial de la articulación y mayoritariamente de forma bilateral. Se observa una influencia negativa en la tasa de caídas manifestadas por el toro durante la lidia, lo que hace necesario nuevas investigaciones y estudios dirigidos a determinar sus posibles causas: factores genéticos, nutricionales y los inadecuados protocolos de entrenamiento entre otras.
\end{abstract}

PALABRAS CLAVE: Toro, Lidia, Caída, Osteocondrosis.

\begin{abstract}
Osteochondrosis is a degenerative process of the joint surfaces. It is a disease of animal development widely described in the horse, with few references in cattle and virtually non-existent in the fighting bulls. The aim of this study was to record the prevalence of osteochondrosis in bulls and steers considering their possible causes and analysing the impact of this disease on the mobility of the animal during the fight. One hundred and twenty (120) fighting bulls, 3, 4 and 5 yr old fought at different bullrings were studied. During the dressing of cattle after fighting a macroscopic study of the carpal-metacarpal joint was performed, for studying the presence of injury in the joint faces involved and their severity. Alongside the fight, each animal was video recorded in order to analyse his mobility and evaluation of the falling syndrome. The results reflect articular cartilage damage in varying degrees in about $80 \%$ of the animals studied, appearing in $63 \%$ of cases on the medial aspect of the joint and mostly bilaterally. A negative influence was observed in the rate of falls expressed by the bull during the fight, and further research will be recommended and studies aimed at determining possible causes: nutritional alterations and inadequate training protocols.
\end{abstract}

KEY WORDS: Bull, Fight, Falls, Osteochondrosis.

Recibido el 19 de octubre de 2016. Aceptado el 2 de marzo de 2017.

a Departamento de Producción Animal. Facultad de Veterinaria de León. Universidad de León. Campus de Vagazana s/n 24071 León, España.

*Autor de correspondencia: jmlomp@unileon.es 
La osteocondrosis es un trastorno degenerativo del cartílago ${ }^{(1)}$ descrito en humanos, en $\operatorname{cerdos}^{(2)}$, perros $^{(3)}$, gatos ${ }^{(4)}$, y caballos $^{(6,7,8)}$. Es considerada como la causa más importante de debilidad en los miembros posteriores en los $\operatorname{cerdos}^{(9)}$, y causa frecuente de cojeras en caballos jóvenes de deporte $^{(5)}$ y en perros ${ }^{(10)}$.

La etiología de la osteocondrosis ha sido objeto de investigación en los últimos años, sin embargo, no existe consenso sobre la definición de la enfermedad y su patogenia, en particular sobre la formación de las lesiones primarias. Sin embargo, la información obtenida actualmente refleja procesos degenerativos y regenerativos secundarios, en lugar de los procesos primarios ${ }^{(11)}$.

La lesión primaria de osteocondrosis articular se define como una "necrosis isquémica focal del cartílago de crecimiento, iniciado por la falta de aporte sanguíneo de los canales del cartílago"(12). Se trata de un fallo focal de osificación endocondral, es decir, una zona del cartílago de crecimiento que no puede someterse a la calcificación de la matriz o invasión vascular, y por tanto no se convierte en hueso(13).

En casos más avanzados, el cartílago persistente es suave y rojizo, y la médula ósea subyacente es edematosa e hiperémica. La lesión se caracteriza histológicamente por un área focal de necrosis que se limita al cartílago de crecimiento y no implica el cartílago articular que cubre el hueso subcondral subyacente. La bibliografía clasifica la enfermedad en tres grados de lesión, de menor a mayor gravedad: osteocondrosis latente, caracterizada por la presencia de un área focal de necrosis del cartílago que se limita al cartílago epifisario; osteocondrosis manifiesta, con la presencia de un fallo focal de osificación endocondral, visible en el examen macroscópico; y osteocondrosis disecante en los estados avanzados, donde se forma una fisura en la zona de necrosis del cartílago y se extiende a través del cartílago $\operatorname{articular}^{(12)}$.

La osteocondrosis ha sido señalada por diferentes autores como una de las causas predisponentes en el desarrollo de caídas de la raza bovina de lidia(14-18). El padecimiento de pérdidas transitorias de la estación y del equilibrio, englobado bajo el término de "síndrome de caída" ha sido estudiado por distintos autores ${ }^{(19)}$, sin identificar una causa única sino un problema multifactorial, donde intervienen factores predisponentes, como la dotación genética, las características del transporte, las exigencias físicas de la lidia, la falta de gimnasia funcional, deficiencias alimenticias, etc. y otros factores determinantes, como circulatorios, nerviosos, metabólicos, endocrinos, etológicos o lesiones ${ }^{(20)}$. En este último grupo entraría el padecimiento de osteocondrosis, el cual ha sido estudiado en este trabajo. El objetivo del mismo fue informar sobre la afectación de esta patología en los cartílagos articulares de carpo y metacarpo de machos de la raza bovina de lidia, y analizar la posible repercusión de esta lesión en la movilidad del animal durante la lidia.

Se estudiaron 120 animales de la raza bovina de lidia, machos de 3 años $(n=24), 4$ años $(n=58)$ y 5 años $(n=38)$, lidiados en cuatro diferentes plazas de toros. Durante el faenado de las reses posterior a la lidia, se realizó un estudio macroscópico de las articulaciones carpometacarpianas, mediante el cual se identificó la presencia de lesiones, evaluando las superficies articulares de los huesos distales carpales (Ossa carpi) C2-3, y superficie articular proximal del hueso metacarpiano (Ossa metarcarpalia) M3-4. Se midió a su vez el diámetro y profundidad de las lesiones, tomando fotografías de cada muestra recogida.

Previamente se obtuvo información sobre la edad del animal, su peso, y las duraciones tanto del periodo de alimentación intensiva como de la fase de entrenamiento físico previos a la lidia.

La caracterización de las lesiones se realizó siguiendo los criterios y metodología descritos en trabajos anteriores ${ }^{(18,21,22)}$ que clasifican las lesiones en 4 grados:

Normal (I): cuando las caras o superficies articulares no mostraban alteraciones macroscópicamente. Leve (II): cuando la lesión fue menor del $20 \%$ de la superficie articular y la escoriación es superficial. Moderada (III): cuando la lesión superó el $20 \%$ de la superficie articular, con pérdida focal del cartílago. Grave (IV): erosión profunda con afectación ósea. 
Paralelamente se grabó la lidia de cada animal para el análisis de su movilidad y la posible claudicación de los animales durante la misma. Para la valoración del síndrome de caída se utilizó la metodología descrita por Alonso et ak ${ }^{(19)}$, que consideran seis tipos diferentes en virtud de la gravedad de la claudicación evidenciada por el animal:

Tipo 1. Caracterizado por una locomoción irregular, así como por el contacto momentáneo de la cara dorsal de la pezuña o la zona articular del menudillo con el suelo.

Tipo 2. Se caracteriza por la flexión momentánea durante el apoyo de la articulación carpo-metacarpo o tarso-metatarso, existiendo o no contacto de dichas articulaciones con el suelo.

Tipo 3. Se produce cuando hay un contacto transitorio con el suelo, durante menos de $10 \mathrm{seg}$, bien del esternón, papada o cabeza, o bien del corvejón, flanco o nalga, según se trate de las extremidades torácicas o pélvicas respectivamente.

Tipo 4. Tiene lugar cuando el animal adopta una posición de decúbito lateral total o esternoabdominal, siempre que su duración sea inferior a 20 seg; igualmente se llega a este tipo de caída cuando en una de tipo 3 el contacto con el suelo tiene una duración superior a 10 seg e inferior a 20.

Tipo 5. A esta variedad de caída se llega cuando el decúbito del animal (caída de tipo 4), o el contacto con el suelo que origina el tipo 3, se prolongan más allá de los 20 seg, pero sin llegar a los 120.

Tipo 6. Se produce este tipo cuando el decúbito tiene una duración superior a $120 \mathrm{seg}$.

A su vez se consideró interesante analizar el momento de presentación de las caídas, es decir, la distribución de las caídas por tercio de lidia en función del tipo de lesión. Por último se elaboró un análisis de correlación entre el peso, la edad y los tiempos de cebo y de entrenamiento con el tipo de lesión. Para el análisis se utilizó el paquete estadístico SPSS para Windows (c) (versión 15.0). Se realizó un análisis descriptivo, un análisis de correlación y el correspondiente ANOVA.
Figura 1. Porcentajes de acuerdo al grado de lesión en toros de lidia

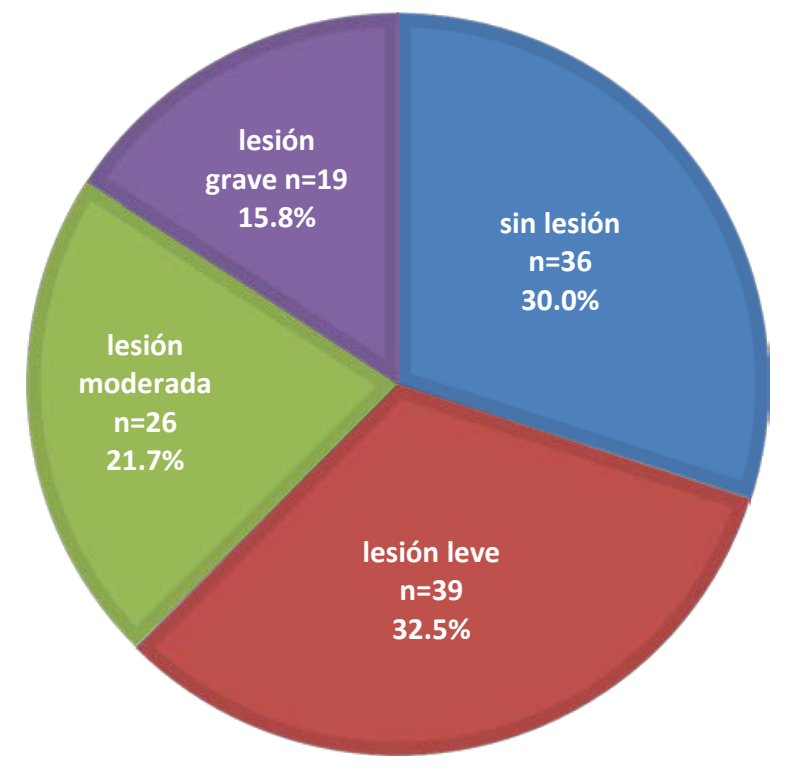

Se encontraron lesiones en el $70 \%$ de los animales muestreados (84 individuos), generalmente de forma bilateral en el $78.3 \%$ de los individuos afectados, siendo más graves las lesiones encontradas en la parte medial de la articulación (Figura 1). Se registraron 36 animales (30 \%) sin ninguna lesión articular (grado I), 39 animales (32.5\%) con lesiones leves (grado II), 26 individuos (21.7\%) con lesiones moderadas (grado III) y 19 animales (15.8 \%) con lesiones graves (grado IV).

El alto porcentaje de aparición de las lesiones en los animales muestreados evidencia que la osteocondrosis es una lesión a tener muy en cuenta en esta raza de bovinos. El hecho de que se haya observado de forma bilateral en el $78.3 \%$, podría apuntar a una causa sistémica, tal y como lo describen diversos autores en $\operatorname{cerdos}^{(13)}$, en perros $^{(23)}$ y en humanos ${ }^{(24,25)}$.

El estudio macroscópico de las articulaciones (Figura 2), mostró una lesión más acusada en la superficies articular de M3-4, en comparación con la cara distal de C2-3, donde es menos evidente. Las lesiones leves, (grado II) están bien delimitadas, por un engrosamiento moderado de los bordes, en su mayoría hiperémicos, el fondo de la lesión, suele 
Figura 2. Imágenes de los diferentes grados de lesión (I, II, III y IV)

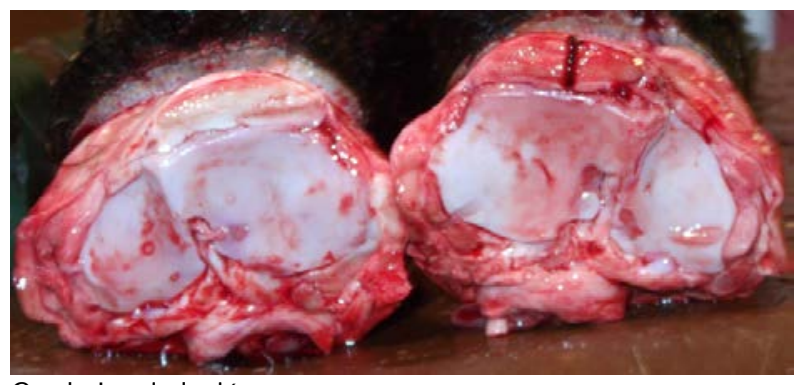

Grado I - sin lesión

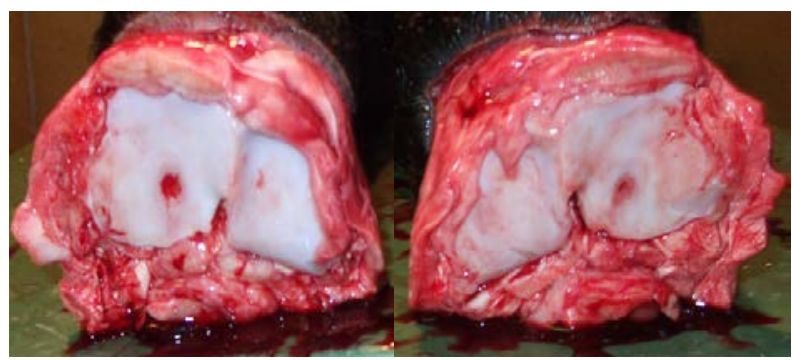

Grado II - leve

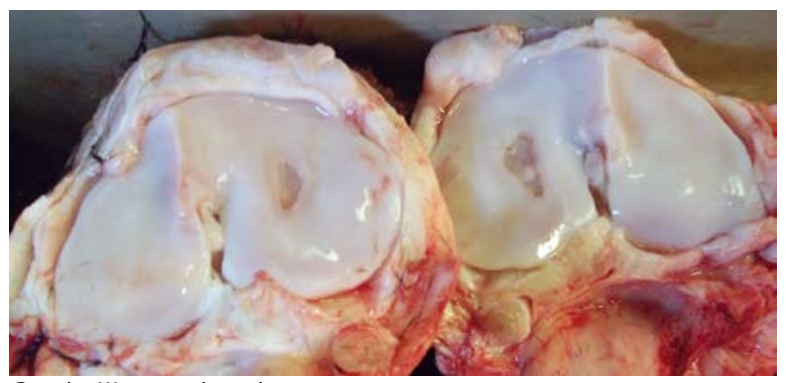

Grado III - moderada

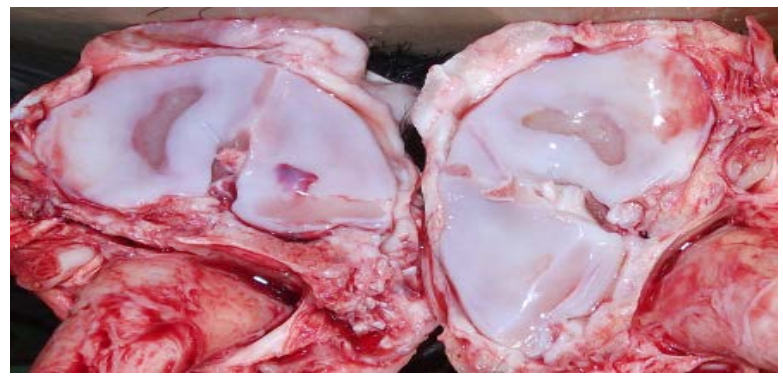

Grado IV - grave

tener una coloración blanquecina y cuando la lesión muestra un proceso agudo, se observa un fondo hiperémico.

Las lesiones moderadas (grado III) mostraron una pérdida de continuidad total de la superficie articular, más acentuada en M3-4, con un borde bien definido, que van desde la coloración blanquecina- nacarada a roja (hiperemia) con ulceración muy evidente. Se observaron restos de desprendimiento cartilaginoso y colgajos sobre dichas lesiones. La dimensión de las lesiones tenía una media de $0.6 \mathrm{~cm}$ de diámetro y $3 \mathrm{~mm}$ de profundidad.

Las lesiones graves (grado IV), estaban muy bien definidas, con bordes redondeados, de forma simétrica y en ambas caras de las superficies articulares correspondientes. De coloración blanquecina y roja (hiperemia); el fondo de las lesiones presentaron una coloración rojiza mostrando en su totalidad el hueso subcondral; el diámetro de las lesiones fue $1.3 \mathrm{~cm}$ de media con una profundidad media de $3 \mathrm{~mm}$.

La localización de las lesiones observadas coinciden con las lesiones descritas en la raza de lidia por otros autores ${ }^{(15,16)}$. Lesiones similares han sido descritas anteriormente en perros ${ }^{(26)}$, en ganado bovino de raza Brahman (27), en caballos de Pura Raza Inglés ${ }^{(5,28)}$ y en $\operatorname{cerdos}^{(12,13)}$, todas ellas observadas en animales con una historia clínica compatible con osteocondrosis y evidenciadas en la necropsia.

Sin embargo, respecto al grado de lesión, se encontró un porcentaje menor de animales afectados en comparación con anteriores trabajos con ganado de lidia(16,17,18). Mientras Dávila(18) observó un $80 \%$ de novillos lesionados, en el presente estudio se encontró la lesión en el $70 \%$ de los individuos. En el caso de los toros (animales de 4 y 5 años) el $73.96 \%$ de los animales mostraron grado de lesión, ligeramente inferior a las encontradas por Mas et a ${ }^{16)}: 86.86 \%$ y Dávila(18): $86.5 \%$. Los resultados de este estudio tampoco coincidieron con ninguno de los trabajos realizados con ganado bravo en cuanto al grado de lesión más frecuente en los animales de 4 y 5 años. En el presente estudio, la lesión leve fue la más común (32.5\%) mientras que para Dávila(18) fue la grave (51 \%) y Mas et al ${ }^{(17)}$ por su parte señalan un $45.3 \%$ de lesiones graves.

Analizando los animales por edades (Figura 3) se observa una tendencia al agravamiento de las lesiones observadas a medida que aumenta la edad. Durante los 3 y 4 años de vida las lesiones son fundamentalmente leves $(29.2 \%$ y $39.7 \%$ respectivamente), mientras que en animales de 5 años la lesión más frecuente fue la moderada, con un aumento significativo de las lesiones graves. Esto 
Figura 3. Grado de lesiones en toros de lidia de acuerdo a la edad (\%)

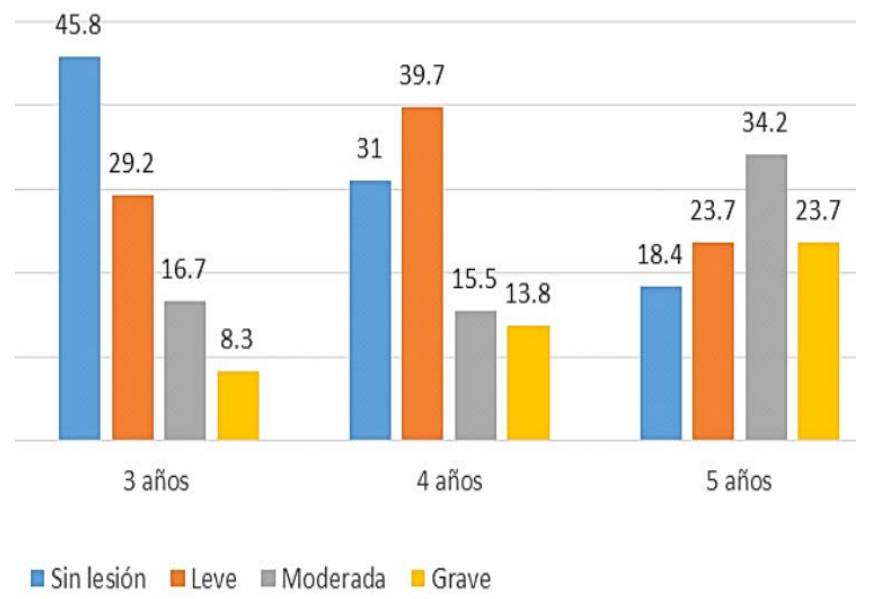

sugiere que las lesiones podrían agravarse con la edad, que viene acompañada de una alimentación intensiva, generando un aumento exponencial de la grasa y la musculatura y por tanto del peso del animal, lo que podría influir de forma directa en la formación de lesiones.

De hecho, en la descripción de su etiología multifactorial $^{(13,29)}$, se incluyen estos factores además de la heredabilidad, las características anatómicas, los traumatismos y un defecto en la vascularización del cartílago epifisario.

En perros se ha informado que un rápido crecimiento aumenta la incidencia de enfermedades óseas, incluyendo la osteocondrosis ${ }^{(30)}$, teoría que se podría aplicar al crecimiento acelerado del toro de lidia durante los 10-12 meses previos a la lidia a través del cebo intensivo al que es sometido.
Igualmente en caballos, se ha observado que un aumento de la insulina y una disminución de la tiroxina circulantes, causadas por las dietas altas en energía, conducen a un fallo de la maduración del cartílago articular e hipertrofia de los condrocitos $^{(31,32)}$. Según esta hipótesis, los condrocitos bajo la influencia de la insulina y la disminución de los niveles de tiroxina persisten como núcleos de cartílago; estos, posteriormente se necrosan cuando son sometidos a factores biomecánicos en la zona de transición entre el cartílago y hueso, produciendo lesiones similares a la osteocondrosis en los $\operatorname{cerdos}^{(32)}$. Sin embargo, estas teorías que podrían aplicarse al cebo del toro de lidia, se contradicen, debido a que la morfología de las lesiones encontradas está caracterizada por la conformación de áreas bien delimitadas de necrosis en el cartílago epifisario ${ }^{(33)}$.

Complementariamente, la genética podría jugar un papel importante predisponente en base a las diferencias observadas en la prevalencia de la osteocondrosis entre diferentes razas de ganado porcino $^{(34)}$, perros ${ }^{(35)}$ y $\operatorname{caballos}^{(28)}$, por lo que el componente hereditario se postula como un factor a estudiar.

Se analizó el número de animales que padecen cada uno de los seis tipos de claudicación (Cuadro 1), observando que el $85 \%$ de los animales ( $n=$ 102) mostró algún signo o manifestación del síndrome. Por otro lado, en ninguno de ellos se apreciaron caídas de tipo 5 y 6 . Se comprobó la existencia de una mayor tasa de caídas tipo 1 y 2 en los animales con lesiones de grado IV con respecto al resto de animales.

Cuadro 1: Tipo de caídas que presenta cada animal en función del grado de lesión y porcentaje de individuos que las presentan

\begin{tabular}{|c|c|c|c|c|c|c|}
\hline Grado de lesión & $n$ & Caída tipo 1 & Caída tipo 2 & Caída tipo 3 & Caída tipo 4 & Caída total \\
\hline 1 & 36 & $0.9 \pm 1.5^{a}$ & $0.9 \pm 1.0^{a}$ & $0.6 \pm 0.8$ & $0.2 \pm 0.4$ & $2.5 \pm 2.1$ \\
\hline II & 39 & $1.1 \pm 1.3^{a}$ & $0.9 \pm 0.9^{a}$ & $0.6 \pm 1.0$ & $0.1 \pm 0.3$ & $3.2 \pm 2.5$ \\
\hline IV & 19 & $1.4 \pm 1.4^{b}$ & $1.3 \pm 0.8^{b}$ & $0.8 \pm 1.7$ & $0.1 \pm 0.3$ & $3.8 \pm 1,2$ \\
\hline Total & 120 & $1.3 \pm 1.5$ & $1.2 \pm 1.3$ & $0.8 \pm 1.1$ & $0.2 \pm 0.4$ & $3.2 \pm 2.6$ \\
\hline$\%$ & & 32.50 & 37.50 & 23.33 & 5.83 & - \\
\hline$P$ & & ** & ** & & & \\
\hline
\end{tabular}

$* *=P<0.001$ 
Este porcentaje total de animales con falta de fuerza fue inferior al registrado por Alonso et $a{ }^{(19)}$, que fue del $99.56 \%$, con toros valorados con el mismo método (software) en las temporadas 19911993, datos que se explican por la alta prevalencia en los años 90 del síndrome de caída ${ }^{(36)}$. Esta disminución del $14.56 \%$ en la tasa de caídas confirma la tendencia de disminución señalada anteriormente por Bartolomé(37), presumiblemente gracias a la gran mejora experimentada en los últimos años del manejo alimentario y sanitario del ganado bravo. Aun así las caídas persisten y es un gran problema a solucionar.

El porcentaje de caída que se registró en el presente estudio está muy por encima del que describen otros autores ${ }^{(38-42)}$. Tal divergencia podría ser explicada no sólo por las fechas o años considerados sino también, por la diferente metodología de evaluación utilizada, que no se basa en el uso de vídeo ni un programa (software) especializado, y no tiene en cuenta las caídas más leves de los animales.

Las variedades de claudicación de tipo 1 y 2 pueden pasar inadvertidas para cualquier espectador, y para todo aquel que no esté pendiente de las extremidades del toro durante su lidia y visualice, de forma repetida, los comportamientos pregrabados pues, estas caídas leves, no suponen una interrupción apreciable del normal discurrir del espectáculo. Las más graves (caídas tipo 3, 4, 5 y 6) sí suponen un problema evidente para la lidia, causando interrupciones que deslucen la faena. En este estudio, sólo el $29.16 \%$ de los animales presentaron estos tipos de claudicación, porcentaje menor al $66.57 \%$ señalado por Alonso et $a^{\left({ }^{19}\right)}$, pero próximo a los señalados por los trabajos anteriormente citados ${ }^{(39,40,41)}$.

Si se toma en cuenta el número de caídas de cada grupo de animales en función de las lesiones encontradas, se observó un mayor porcentaje en los individuos con lesiones grados III y IV, evidenciándose en el caso de las caídas del tipo 1 y 2 (los tipos más frecuentes: $70 \%$ ) para las cuales se encontró diferencias estadísticamente significativas entre los grupos de animales con lesiones de grado III y IV y el resto de animales, lo que pone de manifiesto un efecto negativo de la osteocondrosis en la movilidad del animal.

En cuanto a la distribución de la incidencia de caída por tercios en función del grado de lesión (Cuadro 2), el tercio de muleta es la parte de la lidia donde más caídas desarrollan los animales, siendo significativamente más numerosas las claudicaciones sufridas por los individuos con lesiones grado II, III y IV en comparación con los animales sanos (grado I).

Esta mayor aparición de caídas en la muleta (58.60\%), se encuentra en la misma línea del trabajo de Alonso et $a^{(19)}$, donde las manifestaciones de caída se agravan y aumentan de frecuencia a medida que transcurren los diferentes tercios de la lidia, llegando en la muleta al $50 \%$ de las claudicaciones.

Cuadro 2. Caídas en cada una de las diferentes fases de la lidia en función del grado de lesión y porcentaje de individuos que las presentan

\begin{tabular}{ccccccc}
\hline Grado de lesión & $\mathrm{n}$ & $\begin{array}{c}\text { Caídas } \\
\text { INICIO }\end{array}$ & $\begin{array}{c}\text { Caídas } \\
\text { VARAS }\end{array}$ & $\begin{array}{c}\text { Caídas } \\
\text { BAND }\end{array}$ & $\begin{array}{c}\text { Caídas } \\
\text { MULETA }\end{array}$ & Caída total \\
\hline I & 36 & $0.2 \pm 0.4$ & $0.7 \pm 1.0$ & $0.3 \pm 0.5$ & $1.2 \pm 1.4^{\mathrm{a}}$ & $2.5 \pm 2.1$ \\
II & 39 & $0.4 \pm 0.6$ & $0.6 \pm 0.9$ & $0.4 \pm 0.8$ & $1.9 \pm 2.1^{\mathrm{b}}$ & $3.2 \pm 2.5$ \\
III & 26 & $0.3 \pm 0.7$ & $0.7 \pm 1.1$ & $0.2 \pm 0.6$ & $2.1 \pm 2.3^{\mathrm{b}}$ & $3.5 \pm 2.9$ \\
IV & 19 & $0.3 \pm 0.5$ & $0.5 \pm 0.6$ & $0.3 \pm 0.5$ & $1,8 \pm 1.8^{\mathrm{b}}$ & $3.2 \pm 1,9$ \\
$\mathrm{t}$ & 120 & $0.3 \pm 0.6$ & $0.7 \pm 1.0$ & $0.3 \pm 0.6$ & $1.8 \pm 2.1$ & $3.2 \pm 2.7$ \\
$\%$ & & 8.33 & 22.50 & 10.00 & 75.83 & - \\
$\mathrm{n}$ & & 10 & 27 & 12 & 91 & - \\
\hline
\end{tabular}

$\mathrm{BAND}=$ banderillas.

ab $(P<0.05)$. 
Los animales en los primeros tercios se mueven a mayor velocidad que en fases sucesivas, y en un modelo de ejercicio tipo intermitente, recuperan fuerzas durante los amplios descansos entre desplazamientos ${ }^{(43)}$. En cambio, durante el tercer tercio, el de mayor duración, el toro alterna desplazamientos en serie de largo recorrido con pequeños descansos que no le permiten recuperar. Este tipo de movimiento de cabeza baja, durante el $45.6 \%$ del tiempo de la duración del tercio, predispone al animal a sufrir caídas de tipo 1, 2 y $3^{(44)}$. Además, el animal acumula en este momento un estado elevado y progresivo de fatiga, evidenciado por la abertura de la boca y el aumento de la frecuencia respiratoria ${ }^{(44)}$.

El análisis de las frecuencias de caída por grupos refleja diferencias entre los animales sin lesión (grado I) y los lesionados (grados II, III y IV) en el número de caídas totales en el tercio de muleta, siendo éstas significativamente mayores en los animales con lesión articular. Resultados que fortalecen la teoría de la osteocondrosis como una de las causas predisponentes del síndrome de caída de la raza de lidia ${ }^{(14,15)}$.

Por último, el análisis de correlación del peso de los animales con los diferentes grados de lesión del cartílago articular (Cuadro 3) revela una correlación negativa del mismo con la ausencia de lesión (grado I). Diversos autores ${ }^{(45,46,47)}$ opinan que la caída del toro de lidia, es debida al desarrollo incompleto del aparato locomotor del animal debido a una subnutrición en los primeros años de crecimiento. Por otro lado, se han observado lesiones de osteocondrosis ya desde los primeros 12 meses de vida ${ }^{(18)}$. Esas lesiones tempranas podrían ir paulatinamente intensificándose con la edad y a medida que el peso aumenta con el cebo intensivo previo a la lidia. Como consecuencia del mismo, se produce una mayor fricción en las articulaciones y la lesión podría aumentar en superficie y gravedad.

Por su parte Lomillos(48) obtuvo una correlación positiva con el peso y las caídas del toro durante la lidia, lo cual concuerda con los resultados del presente estudio y la hipótesis de otros autores ${ }^{(17)}$, consistente en que un mayor peso del animal lo predispone a sufrir osteocondrosis. Este sobrepeso a su vez genera una mayor presión sobre las articulaciones y ésta, durante los desplazamientos en la lidia provoca un dolor más fuerte ocasionando mayor número de caídas en el ruedo.

Por su parte la edad y los meses de cebo se correlacionan positivamente con el padecimiento de lesiones de grado II y III. Esto se puede explicar con el hecho que los animales de mayor edad ( 4 y 5 años) son los que siguen un periodo de acabado con una alimentación más intensiva y un entrenamiento que va de 3 a 9 meses, y reflejan una mayor incidencia de este tipo de lesiones. Por el contrario los animales lidiados de menor edad (novillos de 3 años) no siguen una alimentación tan forzada ni protocolos de entrenamiento.

El cambio de una alimentación básicamente extensiva, hacia una alimentación intensiva pre-lidia basada en dietas ricas en carbohidratos durante el último año de vida del animal, conlleva a desequilibrios nutricionales como lo son una suplementación de calcio-fósforo, una deficiencia de cobre, un excesivo aporte de zinc y una deficiencia de vitamina C, A, D, y biotina, propuestos como factores etiológicos de la osteocondrosis ${ }^{(49)}$. Además este cebo intensivo desemboca en un aumento repentino de la grasa y masa muscular, que no va acompañado de un crecimiento esquelético, lo cual puede afectar a la movilidad del animal. De acuerdo

Cuadro 3. Correlación lineal del grado de lesión con el peso, la edad y la duración de cebo y entrenamiento para el conjunto de los animales

\begin{tabular}{lcccc}
\hline Grado de lesión: & $\mathrm{I}$ & $\mathrm{I}$ & $\mathrm{II}$ & $\mathrm{IV}$ \\
\hline Peso, kg & $-0.229^{\star}$ & 0.031 & -0.057 & -0.031 \\
Edad, meses & -0.079 & $0.231^{\star}$ & $0.102^{\star}$ & -0.176 \\
Alimentación intensiva, meses & 0.084 & $0.257^{\star}$ & $0.152^{\star}$ & 0.043 \\
Entrenamiento, meses & -0.150 & 0.043 & $0.255^{\star}$ & $0.183^{\star}$ \\
\hline
\end{tabular}

$*=P<0.05$. 
con ello, en los resultados del presente estudio se observa que la duración de la fase de alimentación intensiva se correlaciona positivamente con las lesiones de grado II y III.

Igualmente, la correlación positiva que se observa entre los meses de entrenamiento de los animales y la aparición de lesiones de grado III y IV (las más severas), hace pensar que la gimnasia funcional podría influir en el agravamiento de las lesiones que podrían haberse iniciado durante el periodo de alimentación intensiva. Este entrenamiento suele practicarse durante los seis meses anteriores a la lidia, y se basa generalmente en forzar a los animales a realizar un ejercicio intenso de carrera durante 30 min, tres veces por semana, lo que implica una mayor fricción en las articulaciones y podría agravar la lesión, incluso producir una cojera(48). Los traumatismos han sido una de las causas más ampliamente citadas como causa de osteocondrosis en todas las especies ${ }^{(1,13)}$ y en sintonía con ello se puede pensar que el entrenamiento es a menudo una fuente de traumas en las articulaciones de los toros y novillos. Generalmente la gimnasia se realiza en los caminos que comunican los diferentes cercados de la ganadería, y el terreno no está adaptado al esfuerzo intenso que realizan los animales, siendo pisos duros. En ocasiones el ganadero prepara el "corredero" con tierra blanda, lo cual tampoco soluciona el problema con los riesgos de distensiones articulares(48). La localización de la lesión tiende a estar situada en puntos de estrés biomecánico local, como es el caso de la articulación del menudillo, además, el aumento del estrés parece incrementar la prevalencia y la gravedad de las lesiones macroscópicas ${ }^{(1)}$, como es el caso de todos los movimientos de animales por la finca para los distintos manejos.

De igual forma, el efecto traumático del entrenamiento podría desembocar en la conversión de una lesión articular subclínica a una osteocondrosis manifiesta o desecante ${ }^{(48)}$, la severidad del trauma por lo general es mínima, y a menudo incluye sólo las fuerzas que intervienen en la ambulación normal. Así, aunque los traumatismos graves se han documentado como causa de fracturas osteocondrales, particularmente en los seres humanos, su papel en el desarrollo inicial de osteocondrosis parece ser limitada, como lo demuestra la falta de una historia que incluye un impacto único y traumático en la mayoría de los animales y humanos ${ }^{(23,24,25)}$. Una lesión traumática continua, como es el entrenamiento, podría explicar el hecho de que las lesiones se localizan en los sitios de predilección específica (menudillo) y, generalmente aparecen de forma bilateral.

\section{CONCLUSIONES E IMPLICACIONES}

Se identifica la osteocondrosis como una lesión común en los machos de la raza de lidia, con una incidencia del $70 \%$ de los animales muestreados, de forma bilateral en el $78.3 \%$ de los individuos afectados, siendo más graves las lesiones encontradas en la cara medial de la articulación. El $85 \%$ de los animales manifestaron caídas de diferente grado, apreciando una mayor tasa de caídas tipo 1 y 2 sufridas por los animales con lesiones de grado IV frente a las experimentadas por el resto de animales, siendo el tercio de muleta la parte de la lidia donde más caídas desarrollan los animales, significativamente más numerosas en los individuos con lesión, debido al mayor esfuerzo de locomoción que desarrolla el animal en esta fase. En base a los resultados del análisis de correlación y la literatura consultada se puede inferir que existen varios factores que podrían predisponer la presentación de lesiones del cartílago articular, como son el aumento de peso debido al cebo intensivo previo a la lidia, la duración del mismo y del protocolo de entrenamiento seguido.

\section{LITERATURA CITADA}

1. Bohndorf K. Osteochondritis (osteochondrosis) dissecans: a review and new MRI classification. Eur Radiol 1998;(8):103-112.

2. Dewey $C$. Diseases of the nervous and locomotor systems. In: Diseases of swine. Straw B, et al. editors, 8th ed. Oxford, UK: Blackwell Science; 1999:861-883.

3. Morgan JP, Wind A, Davidson AP. Enfermedades articulares y óseas hereditarias del perro. República Argentina: Editorial Intermédica; 2001.

4. Ralphs, SC. Bilateral stifle osteochondritis dissecans in a cat. J Am Anim Hosp Assoc 2005; (41): 78-80. 
5. Mcl lwraith, CW. Diseases of joints, tendons, ligaments, and related structures. In: Adams' lameness in horses, Stashak TS editor. 5th ed. Baltimore USA: Lippincott Williams \& Wilkins; 2002:459-644.

6. Jensen R, Park RD, Lauerman LH, Braddy PM, Horton DP, Flack, $\mathrm{DE}$, et al. Osteochondrosis in feedlot cattle. Vet Pathol 1981; (18):529-535.

7. Trostle SS, Nicoll RG, Forrest LJ, Markel MD. Clinical and radiographic findings, treatment, and outcome in cattle with osteochondrosis: 29 cases (1986-1996). J Am Vet Med Assoc 1997:211(12): 1566-1570.

8. Tryon KA, Farrow CS. Osteochondrosis in cattle. Vet Clin North Am Food Anim Pract 1999; 15(2):265-274.

9. Jørgensen B, Andersen S. Genetic parameters for osteochondrosis in Danish Landrace and Yorkshire boars and correlations with leg weakness and production traits. Anim Sci 2000; (71):427-434.

10. Harari J, Osteochondrosis. Vet Clin North Am Small Anim Pract 1998; (28):87-94.

11. Bertone AL, Bramlage LR, Mcl lwraith CW, Malemud C . Comparison of proteoglycan and collagen in articular cartilage of horses with naturally developing osteochondrosis and healing osteochondral fragments of experimentally induced fractures. Am J Vet Res 2005; (66): 1881-1890.

12. Ytrehus B, Carlson CS, Ekman S. Etiology and pathogenesis of osteochondrosis. Vet Pathol 2007;(44)429-448.

13. Ekman S, Carlson CS. The pathophysiology of osteochondrosis. Vet Clin North Am Small Anim Pract 1998; (28):17-32.

14. Urquía JJ, Morales J, Durán J M, Carpintero CM, Fernández C, Flores, $\mathrm{B}$, et al. Consideraciones sobre el estudio de la osteocondrosis de la articulación carpo-metacarpiana en el toro de lidia. IV Symposium Nacional del Toro de Lidia. Zafra. 1999:255-258.

15. Dávila U, Méndez J L, Aja S, Calva B, Sierra MA, Téllez JR, Méndez A. Osteocondrosis, alteración patológica influyente en la caída del toro de lidia. Congreso Mundial Taurino de Veterinaria. Murcia (España). 2008:219-223.

16. Mas A, Martínez-Gomariz F, Sanes JM, Gutiérrez C, Motas M, Pallarés FJ. Estudio macroscópico y estructural de la osteocondrosis en la articulación carpometacarpiana en el toro de lidia. Symp Toro Lidia, Zafra (España). 2011:185-188.

17. Mas A, Martínez-Gomariz F, Sanes JM, Sánchez C, Reyes JA, Gutiérrez C, Seva JI. Estudio estadístico de la relación de la edad y el peso con la aparición de osteocondrosis carpometacarpiana y síndrome de la caída en el toro de lidia. Symp Toro Lidia, Zafra (España). 2011:189-193.

18. Dávila U. Osteocondrosis en el toro de lidia [tesis doctoral]. Córdoba, España: Universidad de Córdoba; 2013.

19. Alonso ME, Sánchez JM, Robles R, Zarza AM, Gaudioso VR. Relation entre la fréquence de la chute et différents paramètres hematologiques chez le toreau de combat. Revue de Medecine Vétérinaire 1997;(148)12:999-1004.

20. Martínez P. Lesiones anatómicas producidas en el toro por los trebejos empleados en la lidia [tesis doctoral]. Córdoba, España: Universidad de Córdoba; 1997.

21. Martínez-Gomariz, F, Mas A, Vázquez J M, Gil F, Seva JI, Sánchez C, et al. Estudio artroscópico de la osteocondrosis carpometacarpiana del toro de lidia. VII Congreso Mundial Taurino de Veterinaria. Cáceres (España) 2011:175-180.
22. Fernández FJ, Ortuño S, Recas EA. Osteocondrosis metacarpiana del toro de lidia: primeros resultados comparativos entre los estudios del 2000 y 2009. Symp Toro Lidia, Zafra (España). 2011:99-100.

23. Peterson RK, Savoie FH, Field, LD. Osteochondritis dissecans of the elbow. Instr Course Lect 1999; (48):393-398.

24. Robertson W, Kelly BT, Green DW. Osteochondritis dissecans of the knee in children. Curr Opin Pediatr 2003; (15):38-44.

25. Blitz NM, Yu JH. Freiberg's infraction in identical twins: a case report. J Foot Ankle Surg 2005; (44):218-221.

26. Leighton RL. Historical perspectives of osteochondrosis. Vet Clin North Am Small Anim Pract 1998; (28):1-16.

27. Hill $\mathrm{BD}$, Sutton $\mathrm{RH}$, Thompson $\mathrm{H}$. Investigation of osteochondrosis in grazing beef cattle. Rockhampton Veterinary Laboratory, Queensland. Aust Vet J 1998;76(3):171-175.

28. Grøndale, AM, Dolvik NI. Heritability estimations of osteochondrosis in the tibiotarsal joint and of bony fragments in the palmar/plantar portion of the metacarpo- and metatarsophalangeal joints of horses. J Am Vet Med Assoc 1993;(203):101-104.

29. Schenck RC, Goodnight J M. Osteochondritis dissecans. J Bone J oint Surg Am 1996; (78): 439-456.

30. Richardson DC, Zentek J. Nutrition and osteochondrosis. Vet Clin North Am Small Anim Pract 1998; (28):115-135.

31. Shingleton WD, Mackie EJ, Cawston TE, J effcott LB. Cartilage canals in equine articular/epiphyseal growth cartilage and a possible association with dyschondroplasia. Equine Vet J 1997; (29):360-364.

32. J effcott LB, Henson FM. Studies on growth cartilage in the horse and their application to aetiopathogenesis of dyschondroplasia (osteochondrosis). Vet J 1998;156:177-192.

33. Carlson CS, Cullins LD, Meuten DJ. Osteochondrosis of the articularepiphyseal cartilage complex in young horses: evidence for a defect in cartilage canal blood supply. Vet Pathol 1995;(32):641-647.

34. Van der Wal PG, Van der Valk PC, Goedegebuure SA, Van Essen G. Osteochondrosis in six breeds of slaughter pigs. II. Data concerning carcass characteristics in relation to osteochondrosis. Vet Q 1980; (2):42-47.

35. LaFond E, Breur GJ, Austin CC. Breed susceptibility for developmental orthopedic diseases in dogs. J Am Anim Hosp Assoc 2002; (38):467-477.

36. Castejón FJ. Incoordinación motora y caída del ganado bravo durante la lidia. Bol Inf SYVA. 1985:40-44.

37. Bartolomé DJ. La acidosis del toro de lidia [tesis doctoral]. León, España. Universidad de León; 2009.

38. J ordano D, Gómez-Cárdenas G. Investigaciones sobre la caída de los toros de lidia. Arch Zootec 1954:3(9):3-52.

39. García-Belenguer S. Estudio de degeneraciones musculares en ganado bravo y su relación con la fuerza exhibida por los animales durante la lidia [tesis doctoral]. Zaragoza, España: Universidad de Zaragoza; 1991.

40. Purroy A, García-Belenguer. La falta de fuerza en el toro bravo. El Campo 1992; (125):49-56.

41. Alonso R, Hebrero C, Pizarro M. La caída del toro bravo y su posible relación con el encaste, el peso y la edad. Rev Prof Vet 2006(66):32-34. 
42. Aceña MC. Estudio de la respuesta de estrés en el toro bravo y su relación con la fuerza y la adaptación muscular al ejercicio durante la lidia [tesis doctoral]. Zaragoza, España: Universidad de Zaragoza; 1993.

43. Paniagua FJ. Tiempos de lidia y de ejercicio del toro. Congreso Mundial Taurino de Veterinaria. Córdoba. 1997:143-145.

44. García-Scheider J MN. Développement et validation d'une nouvelle méthode quantitative et objective $d^{\prime}$ 'evaluation du comportement et des dépenses énergétiques du taureau Brave au cours de la corrida [these doctorel]. Toulouse, France: Université Paul-Sabatier de Toulouse; 2008.
45. Mármol M. La caída del toro de lidia. Ganadería 1967(292):533535.

46. Molina J. La caída de los toros de lidia. Ganadería 1968(307):3539.

47. Ruiz L. ¿Por qué se caen los toros? Ganadería 1971(333):141-143.

48. Lomillos J M. Uso de nuevas tecnologías para el estudio de la raza de lidia [tesis doctoral]. León, España: Universidad de León; 2012.

49. Nakano T, Aherne FX. Involvement of trauma in the pathogenesis of osteochondritis dissecans in swine. Can J Vet Res 1988;(52):154155. 